\title{
Supplementary Material: First-principles quantum and quantum-classical simulations of exciton diffusion in semiconducting polymer chains at finite temperature
}

\author{
Rainer Hegger, Robert Binder, and Irene Burghardt \\ Institute of Physical and Theoretical Chemistry, Goethe University Frankfurt, \\ Max-von-Laue-Str. 7, 60438 Frankfurt/Main, Germany \\ Author to whom correspondence should be addressed: burghardt@chemie.uni-frankfurt.de
}

\section{S1. Kinetic energy operator in curvilinear coordinates}

As detailed in Ref. [1], the kinetic energy operator was determined for a dimer species (OT-2) and the results were transposed to larger oligomer species. This procedure is rationalized by the fact that polythiophene chains, representative of the poly(3-hexylthiophene) (P3HT) material, fall into the class of semiflexible polymers with small persistence lengths [2-4]. Experimentally determined values of the persistence length of $\mathrm{P} 3 \mathrm{HT}$ are of the order of $1-3 \mathrm{~nm}$, such that local conformational motions are essentially short-ranged. Hence, OT-2 is a minimal system suitable to determine the kinetic energy operator for our generalized FH model.

The kinetic energy operator $\hat{T}$ in curvilinear coordinates is obtained using the TNum procedure [5]. To start with, a fully non-separable form of $\hat{T}^{\mathrm{OT}-2}$ is set up,

$$
\hat{T}^{\mathrm{OT}-2}=\frac{1}{2} \sum_{\alpha, \beta} \hat{p}_{\alpha} G_{\alpha \beta}\left(x_{1}, x_{2}, y_{1,2}, \theta_{1,2}\right) \hat{p}_{\beta},
$$

where $\alpha$ and $\beta$ refer to the dimer coordinates $\left\{x_{1}, x_{2}, y_{1,2}, \theta_{1,2}\right\}$.

The calculation of the metric tensor $G_{\alpha \beta}\left(x_{1}, x_{2}, y_{1,2}, \theta_{1,2}\right)$ at several geometries along the intra-monomer torsion mode $\theta_{1,2}$ shows that

- $G_{x x} \equiv G_{x_{n} x_{n}}, n=1,2$, is almost constant, and the coupling $G_{x_{1} x_{2}}$ is negligible;

- the couplings between the local modes $x_{n}$ and the torsional mode $\theta_{1,2}$, i.e., $G_{x \theta} \equiv G_{x_{n} \theta_{1,2}}$, $n=1,2$, is negligible, but the coupling $G_{x y} \equiv G_{x_{n} y_{1,2}}, n=1,2$, is non-zero;

- the variation of the metric tensor as a function of the coordinates is small, such that we may assume, to a good approximation, that the $\mathbf{G}$ tensor is constant.

Within these approximations, the form of the $\mathbf{G}$ tensor specified in Eq. (3) and Table 1 of the main manuscript is obtained. 


\section{S2. Construction of monomer potentials by the analytic mapping pro- cedure of Binder et al. (J. Chem. Phys. 141, 014101 (2014))}

Here, we briefly illustrate the workflow of the analytic mapping procedure [6, 8] that was employed to generate the potentials underlying the present simulations. Details of the analytical form of the potentials are summarized in Appendix A.

As illustrated in Fig. S1 for a PES cut along a ring-torsional mode, ground and excited state $a b$ initio data were computed for an octamer species (OT-8); the same data were employed in Ref. [1. In panel a), the first singlet states $S_{n}, n=0, \ldots 4$ are shown. For the mapping procedure, the data for the $S_{0}, S_{1}, S_{2}$ states are selected to yield monomer potentials and couplings by the solution to an inverse eigenvalue problem [6]. These monomer quantities - i.e., $\left(v_{\mathrm{G}}, v_{\mathrm{E}}, w\right)$ - are shown in panel b) of Fig. S1. These exactly reproduce the input data for the $S_{0}, S_{1}, S_{2}$ states when constructing the generalized Frenkel-Holstein Hamiltonian according to Sec. 2.1 of the main text. At this stage, the procedure is still carried out in a pointwise fashion, but smooth potentials can be generated by fitting to analytical form as discussed in Appendix A.

Finally, the monomer potentials are used to generate potentials for a larger OT-20 system (panel c) that is employed in the present work.

When more approximate electronic structure methods are employed, it is feasible to match the system sizes in the mapping procedure and in the dynamical simulations. For example, in our recent studies of oligo( $p$-phenylene vinylene) species [8], TDDFT calculations were carried out for an OPV-20 system, which was subsequently treated in the quantum dynamics.
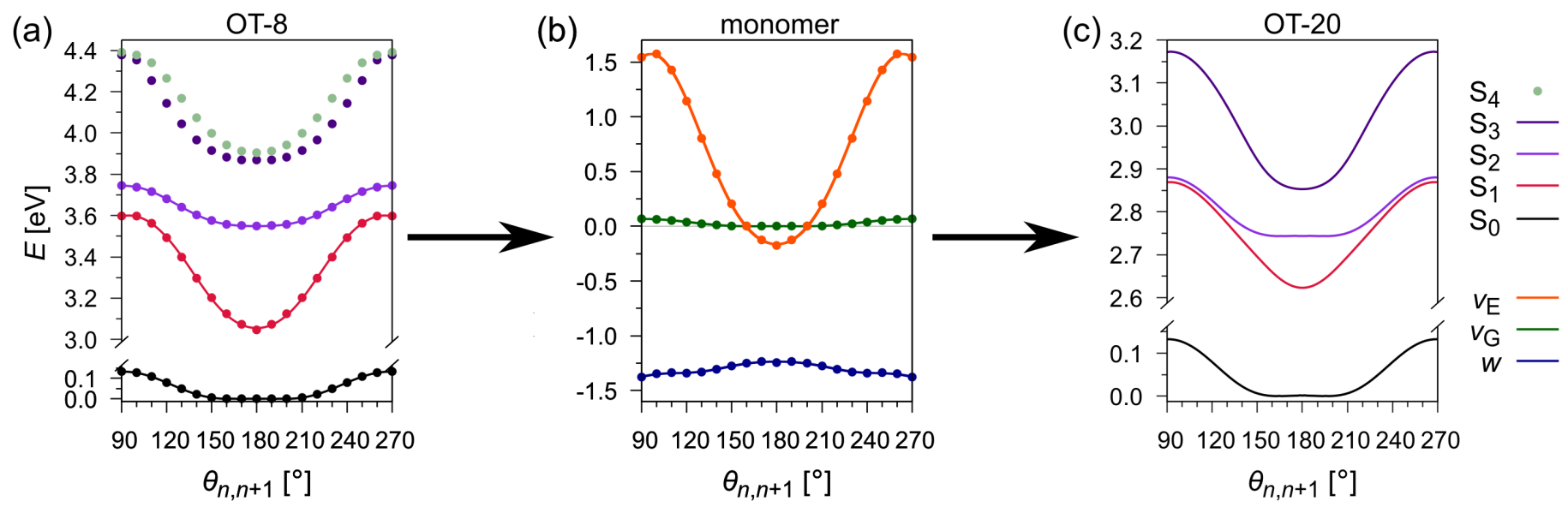

Figure S1 - Workflow of the analytic mapping procedure as applied to the present system: Highlevel ADC(2) electronic structure information computed for an OT-8 system (panel a) is mapped onto a monomer representation (panel b) and subsequently transported to the OT-20 system that is employed in the dynamical simulations.

\section{S3. Torsional ground-state distribution}

Fig. S2 shows the torsional equilibrium distribution in the electronic ground-state $\left(\mathrm{S}_{0}\right)$, as obtained from quantum mechanical calculations based on the eigenstates of the torsional potential (see Fig. 2 of Ref. [7]) and, for comparison, the corresponding distribution obtained from classical MD simulations. As can be seen from the figure, the distributions are in good agreement at $T=100 \mathrm{~K}$ (red trace/circles) and $T=300 \mathrm{~K}$ (blue trace/circles). 
The $\mathrm{S}_{0}$ equilibrium distribution peaks around the planar geometry, but remains non-zero in the regions $\theta<150^{\circ}$ (and $\theta>210^{\circ}$ ), where the excitonic wavefunctions exhibit signatures of conjugation breaks [1]. The likelihood of reaching these areas increases with increasing temperature, as can be seen from the comparison between the distributions at $T=100 \mathrm{~K}$ and $T=300 \mathrm{~K}$.

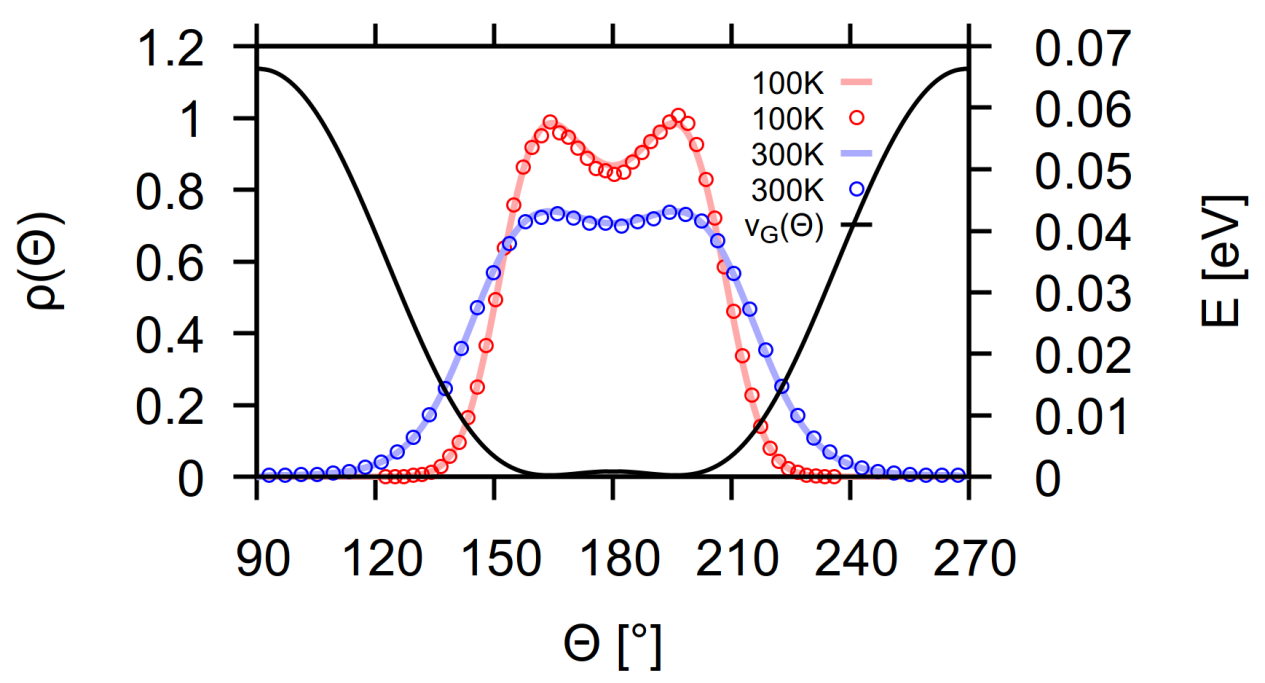

Figure S2 - Torsional distribution in the electronic ground state $\left(\mathrm{S}_{0}\right)$ of the $s$-trans oligothiophene species according to the parametrization described in the main text and in Ref. [1], at $T=100$ $\mathrm{K}$ (red trace/circles) and $T=300 \mathrm{~K}$ (blue trace/circles), obtained quantum mechanically (full lines) as compared with a classical MD based ensemble calculation, using 400,000 trajectories whose torsional distribution is binned (see circles). The torsional potential $v_{G}(\theta)$ is also shown (black trace), see also Fig. 1b) of the main text. Due to the shallow potential barrier at $180^{\circ}$, the torsional distributions exhibit two symmetrically disposed local maxima. At higher temperatures - as already seen at $T=300 \mathrm{~K}$ - these maxima are barely visible, resulting in a wide plateau.

\section{S4. Periodic boundaries: Periodic image representation}

The simulation results presented in the main text have been obtained using periodic boundary conditions (PBCs). Complementary to the representation chosen in the main text, where the physical lattice is shown, we illustrate here the periodic images, for a typical Ehrenfest trajectory. 

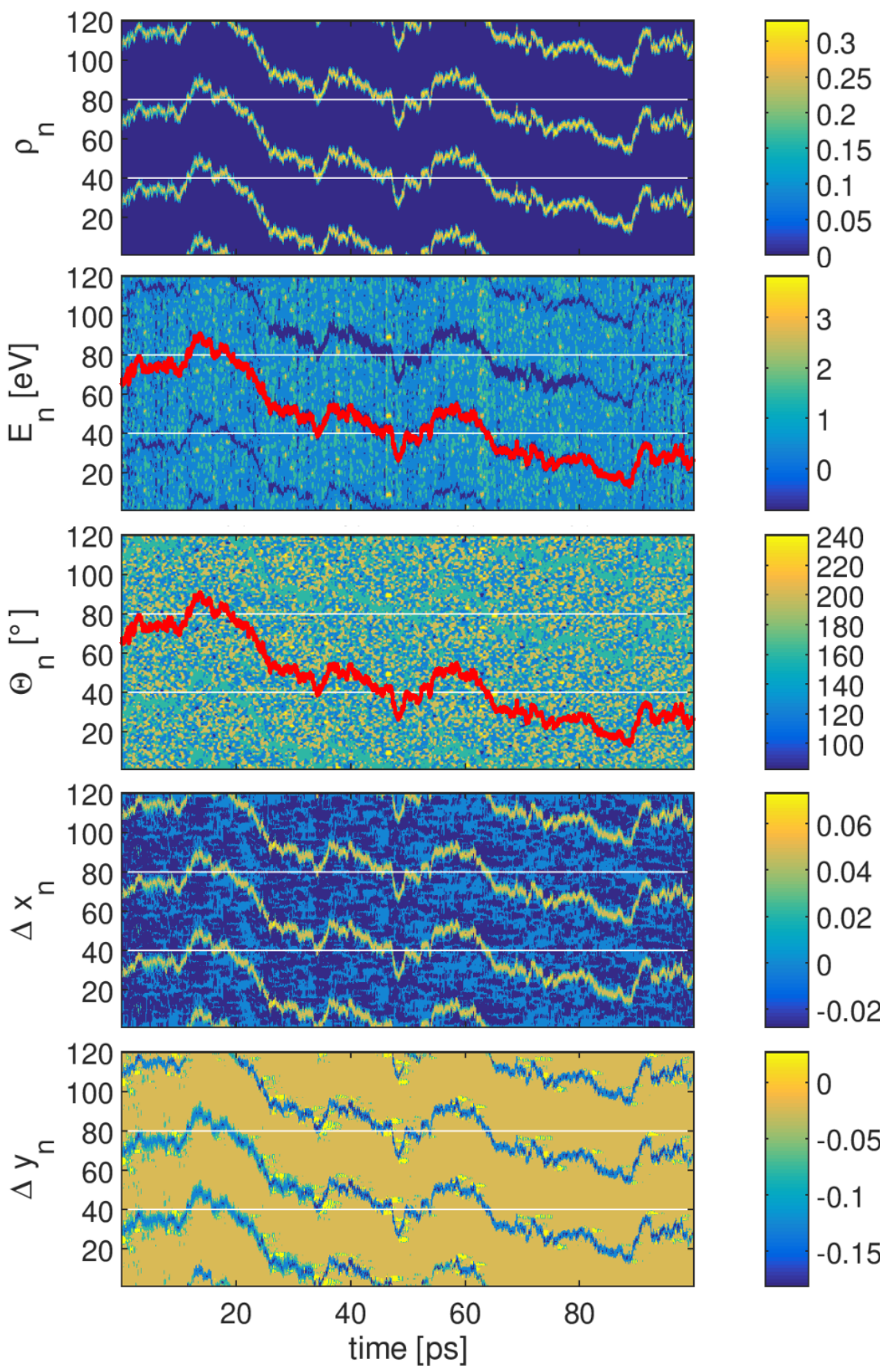

Figure S3 - For an Ehrenfest trajectory on a 40-site lattice using PBCs, we illustrate a set of periodic images. The same quantities are shown as in Figure 2 of the main text.

\section{S5. Origin of the high-frequency oscillations in Ehrenfest simulations}

As pointed out in the main text, all Ehrenfest simulations exhibit spurious high-frequency oscillations - which are not present in the ML-MCTDH reference calculations - that are visible in the exciton density and essentially in all observables.

To understand the origin of these oscillations, we carried out additional simulations where the dynamics is projected onto $S_{1}$. That is, we still carry out the time integration using a diabatic algorithm, in the basis of Frenkel monomer excitations, but project the Ehrenfest 

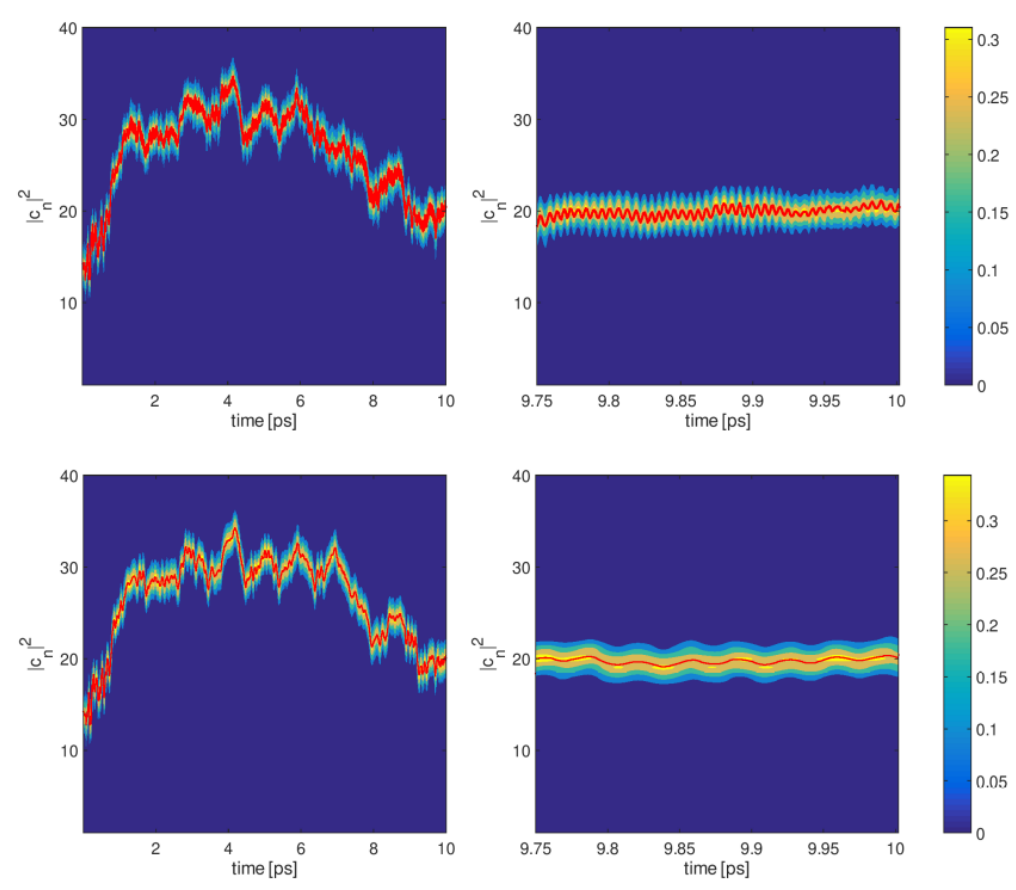

Figure S4 - Comparison between a typical Ehrenfest trajectory (upper panels) and a corresponding trajectory from a dynamics that has been projected onto $S_{1}$. The red traces correspond to the average of the exciton distribution.
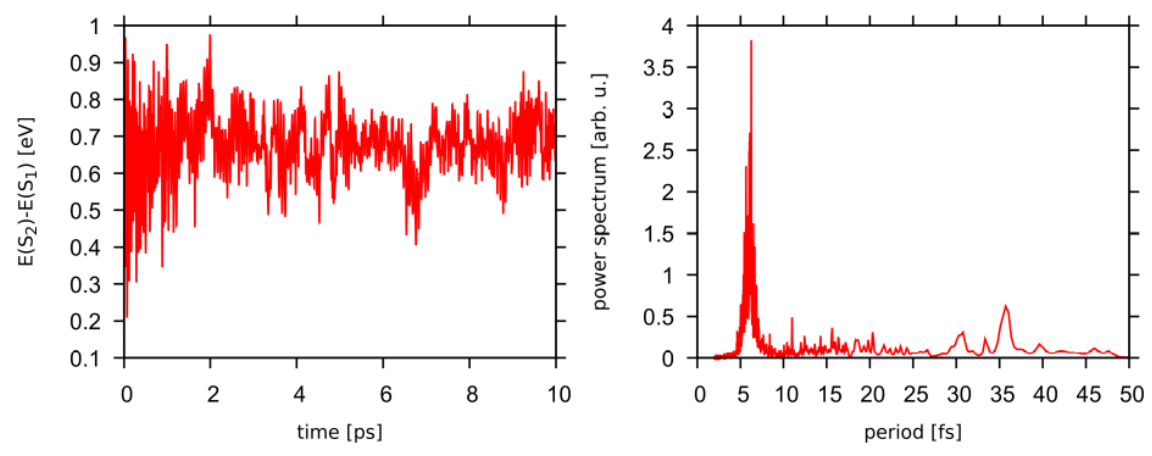

Figure S5 - Left panel: Time-dependent $S_{1}-S_{2}$ gap, Right panel: Power spectrum of the timedependent average exciton population shown in Fig. S4 (upper panels). A high-pass filter was used (moving average with a time window of $20 \mathrm{fs}$ ) prior to Fourier transformation.

wavefunction onto the lowest adiabatic state $\left(S_{1}\right)$ at each point in time. As can be seen from Figure S4 (lower panels), the highest-frequency oscillations disappear in these simulations, and the remaining oscillations are characteristic of coherent vibrational motions of the highfrequency modes (with periodicities around 20-40 fs). Hence, we conclude that the highestfrequency oscillations must be due to electronic coupling between the $S_{1}$ state and higher electronic states, mainly $S_{2}$. This is borne out by inspection of the $S_{1}-S_{2}$ gap in Fig. S5 (left panel), along with the Fourier transform of the time evolving exciton center trajectory (right panel). A peak around 6 fs is clearly visible which indeed corresponds to the average $S_{1}-S_{2}$ gap (around $0.68 \mathrm{eV}$, i.e., a periodicity of $6.1 \mathrm{fs}$ ). Hence, the observed oscillations are essentially due to coherent oscillations between the $S_{1}$ and $S_{2}$ states. 


\section{S6. Finite size effects: lattice size}

The results reported in the main text have been computed, for the most part, for a lattice size of $N=40$, using PBC. Here, we demonstrate that this lattice size is sufficient, by comparing results for the exciton diffusion coefficient at $T=300 \mathrm{~K}$ obtained for $N=10, \ldots, 80$. As can be inferred from Fig. S6, the differences in the diffusion coefficient for these lattice sizes fluctuate in the range of about $1-2 \%$ for $N=20, \ldots, 80$. A qualitatively different behavior is observed for $N=10$, where the diffusion coefficient increases strongly. In this case, the intrinsic size of the exciton is close to the lattice dimension and does not yield a faithful picture of the dynamics. Overall, we may conclude that $N=40$ is an adequate choice for the Ehrenfest simulations using PBC.

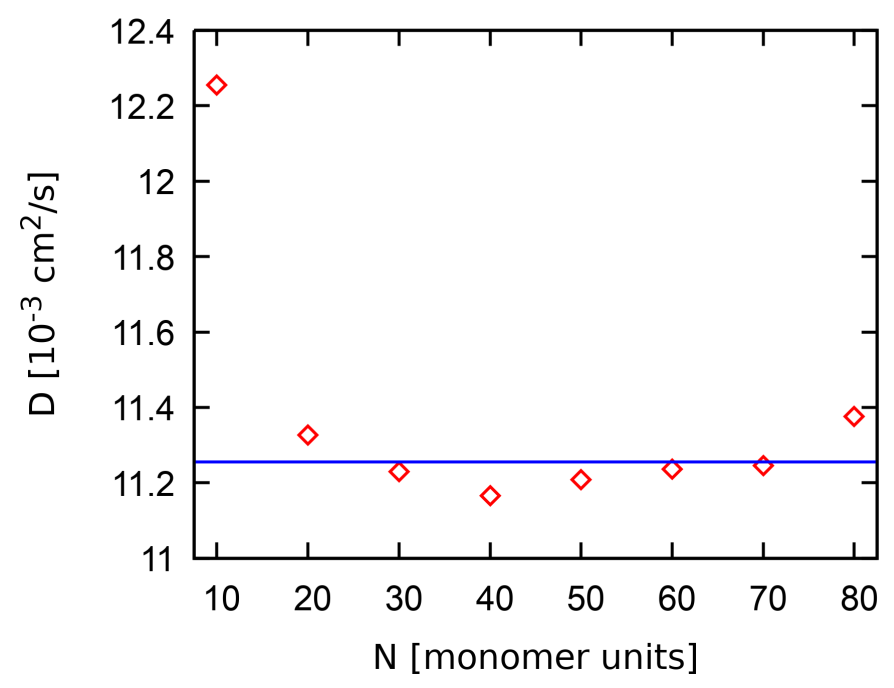

Figure S6 - Computed exciton diffusion coefficient at $T=300 \mathrm{~K}$ as a function of lattice size $N$, in the absence of static disorder.

\section{S7. Convergence of trajectory ensembles}

In view of the high dimension of the classical phase space, one would expect that a large ensemble of initial conditions is necessary to obtain reasonable convergence of the measured quantities. As Fig. S7 indicates, this is not necessarily the case for the computation of the exciton diffusion coefficient. The figure shows the dependence of the diffusion coefficient on the ensemble size, ranging from 500 to 4000 Ehrenfest trajectories, along with the associated standard deviation computed for $10^{3}$ sub-ensembles. These results suggest that (i) the average is quite well converged even for small trajectory ensembles, and (ii) the accuracy steadily improves, i.e., the standard deviation decreases as a function of the ensemble size. The quality of the results may seem surprising since these simulations were performed at $T=300 \mathrm{~K}$, i.e., the highest temperature we studied. For lower temperatures, we would expect even smaller deviations. The reason for the good convergence properties may relate to the fact that, despite the pronounced electronic delocalization effects, the torsional and high-frequeny motions are well described within reduced subspaces of the full phase space. In that sense, the phase space of the extended FH Hamiltonian likely exhibits a "simple" structure. 


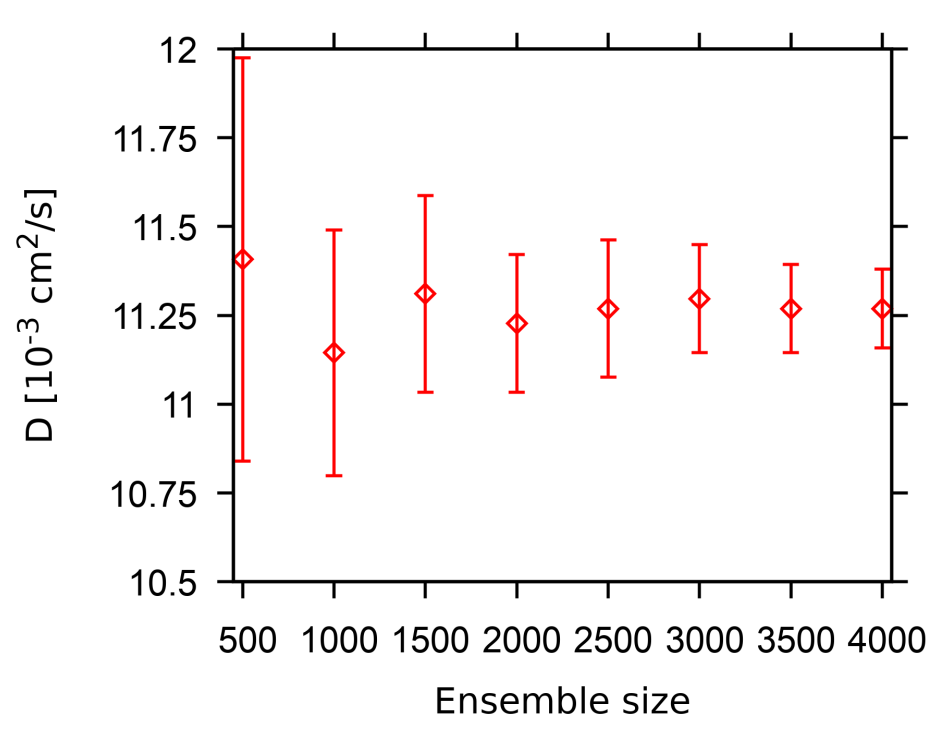

Figure S7 Exciton diffusion coefficient at $T=300 K$ as a function of the trajectory ensemble size, ranging from $K=500$ to $K=4000$ realizations. For each value of $K, L=10^{3}$ sub-ensembles were constructed, such that the diffusion coefficient is obtained as a double average over $K$ and $L$. These double averages are shown as diamonds, while the vertical bars indicate the standard deviation $\pm \sigma_{L}$ with respect to the set of $L$ sub-ensembles.

\section{S8. Finite size effects: Periodic boundary conditions and effect on MSDs}

Since the computation of the diffusion coefficient a priori requires long-time simulations (and, strictly speaking, the diffusion coefficient is defined in the limit $t \rightarrow \infty$ ), finite-size effects will significantly influence the simulation results. In particular, artificial transient effects may arise if the exciton reaches the end of the lattice at some point, see the detailed discussion in the supplementary material of Ref. [6]. Therefore, periodic boundary conditions (PBC) are introduced as discussed in the main text.

Here, we illustrate the effect of PBC on the computed mean squared displacement (MSD) $\langle\Delta \bar{n}\rangle(\Delta t)$ (see Eq. (14) of the main text), from which the diffusion coefficient is obtained by the relation $\langle\Delta \bar{n}\rangle(\Delta t)=2 D \Delta t^{\alpha}$ (see Eq. (16) of the main text). A comparison of MSD computations without vs. with $\mathrm{PBC}$ is shown in Fig. S8, both for quantum dynamical MLMCTDH simulations and for Ehrenfest simulations.

As is seen from the figure, introducing PBC has a major impact on all MSD calculations. In fact, the calculations in the absence of $\mathrm{PBC}$ exhibits a functional form which is not linear, indicating a subdiffusive motion of the exciton. This effect disappears if a lattice with PBC is employed.

For the calculations with PBCs, approximate results for the diffusion coefficients are (i) for the Ehrenfest calculations: $\mathrm{D}=0.7 \times 10^{-2} \mathrm{~cm}^{2} \mathrm{~s}^{-1}(100 \mathrm{~K}), \mathrm{D}=1.05 \times 10^{-2} \mathrm{~cm}^{2} \mathrm{~s}^{-1}(200 \mathrm{~K})$, $\mathrm{D}=1.12 \times 10^{-2} \mathrm{~cm}^{2} \mathrm{~s}^{-1}(300 \mathrm{~K})$, and (ii) for the ML-MCTDH calculations: $\mathrm{D}=1.25 \times 10^{-2}$ $\mathrm{cm}^{2} \mathrm{~s}^{-1}(100 \mathrm{~K}), \mathrm{D}=1.6 \times 10^{-2} \mathrm{~cm}^{2} \mathrm{~s}^{-1}(200 \mathrm{~K}), \mathrm{D}=2.0 \times 10^{-2} \mathrm{~cm}^{2} \mathrm{~s}^{-1}(300 \mathrm{~K})$. This agreement is reasonable, given the different time intervals for which the data were collected. The quantum calculations tend to yield increased values of the diffusion coefficients. 

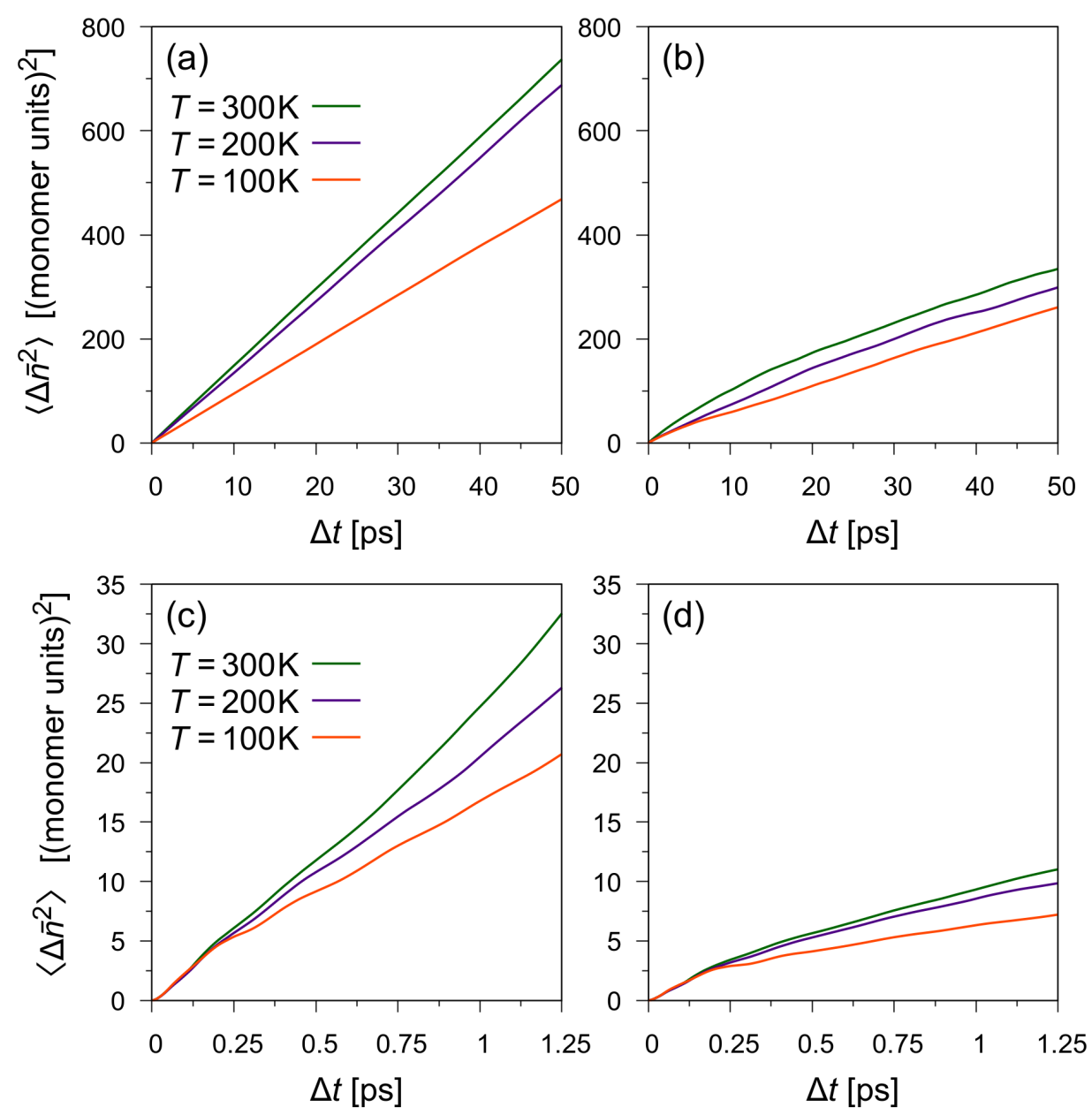

Figure S8 Finite size effects on the MSD. (a) Ehrenfest simulations for an OT-40 system with PBC at $T=100 \mathrm{~K}, 200 \mathrm{~K}$, and $300 \mathrm{~K}$, with the MSD computed up to $50 \mathrm{ps}$; (b) corresponding results for an OT-40 chain without PBC. (c) ML-MCTDH simulations for an OT-20 system with $\mathrm{PBC}$, at $T=100 \mathrm{~K}, 200 \mathrm{~K}, 300 \mathrm{~K}$, with the MSD computed up to $1.25 \mathrm{ps}$, (d) corresponding results for an OT-20 system without PBC.

\section{S9. Adiabatic population analysis as a function of temperature}

For typical Ehrenfest realizations corresponding to $T=50 \mathrm{~K}, 150 \mathrm{~K}, 300 \mathrm{~K}$, for an OT-40 lattice with PBC, Fig. S9 shows the adiabatic populations of the $S_{n}, n=1, \ldots, 4$ states as a function of time. In all cases, the $S_{1}$ population increases during the propagation interval at longer time scales, even though admixtures of higher excited states can be non-negligible at short times. Especially at the initial time, some 10\%-20\% admixture of higher excited states is observed - similarly to our benchmark results for an OT-20 system as reported in Fig. 3(a) of the main text - but nearly $90 \%$ of the population stays in $S_{1}$ on longer time scales even for $T=300 K$. This holds for a majority of trajectories, except for few realizations where larger admixtures of higher excited states occur. In certain cases, the Ehrenfest propagation involves large excited-state contributions and encounters instabilities; this effect is connected to the well-known violation of detailed balance (see discussion in the main text). 
(a)

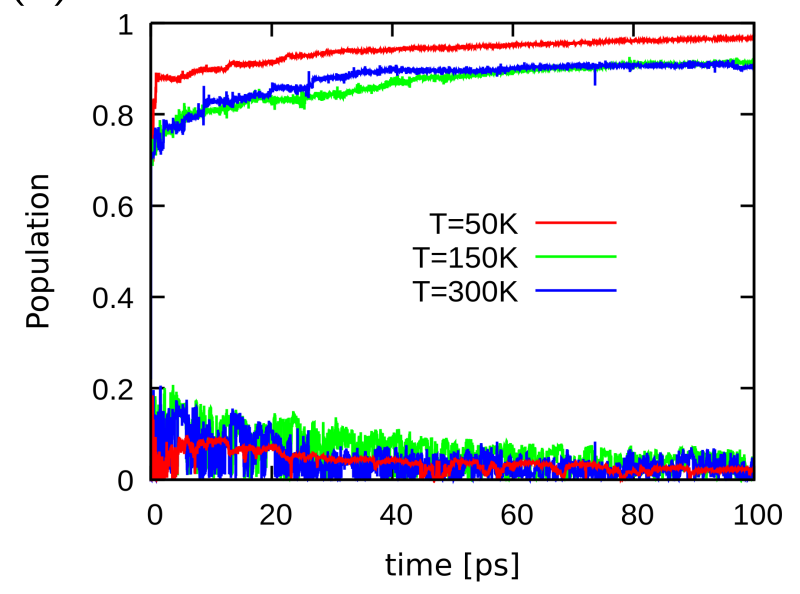

(b)

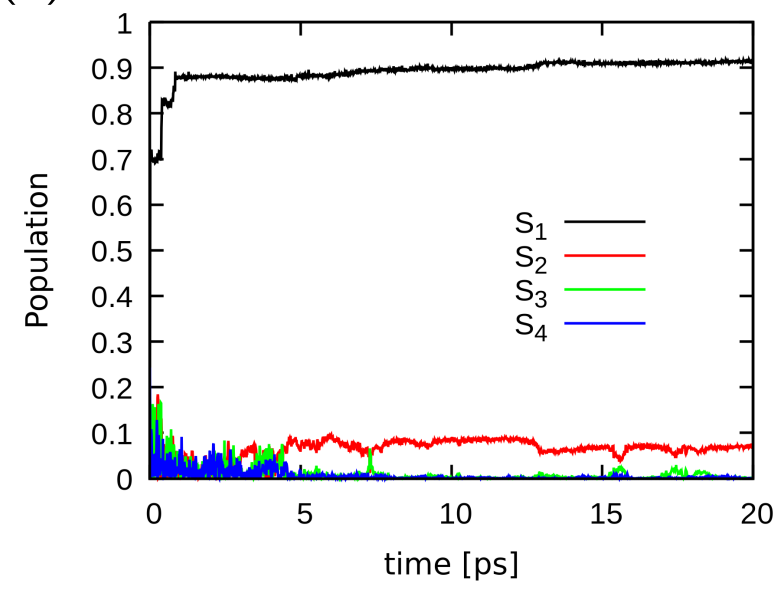

Figure S9 Time-evolving adiabatic populations computed from Ehrenfest simulations for an OT40 system (a) at $T=50 \mathrm{~K}, 150 \mathrm{~K}, 300 \mathrm{~K}$, on a time scale of $100 \mathrm{ps}$, where $\mathrm{S}_{1}$ (upper traces) and $\mathrm{S}_{2}$ (lower traces) are shown, and (b) at $T=50 \mathrm{~K}$, on a shorter time scale of $20 \mathrm{ps}$, where the $\mathrm{S}_{n}, n$ $=1, \ldots, 4$ populations are shown. From both panels, it is seen that initial transients can involve higher excited states, but on longer time scales, the $\mathrm{S}_{1}$ population dominates.

\section{S10. Mean-Squared Displacement (MSD) computation according to Eq. (15)}

Beyond Eq. (14) of the main text, which refers to the MSD of the mean exciton position, Eq. (15) refers to a more general definition that is employed in a quantum setting, such as to account for the time-evolving change of the spatial extension of the exciton. The expression that is usually found in the literature is given as

$$
\left\langle\Delta n^{2}\right\rangle(\Delta t)=\left\langle\sum_{n} \rho_{n}\left(t_{0}+\Delta t\right)\left(n-n_{0}\right)^{2}\right\rangle
$$

where the angular brackets denote an ensemble average (i.e., not a time average) and $n_{0}$ denotes the initially occupied site, for a strictly localized initial condition. Furthermore, if $n_{0}=0$, a simpler expression is obtained,

$$
\left\langle\Delta n^{2}\right\rangle(\Delta t)=\left\langle\sum_{n} \rho_{n}\left(t_{0}+\Delta t\right) n^{2}\right\rangle
$$

In the present context, we augmented the above equations such as to account for the initial delocalization of the excitonic state,

$$
\left\langle\Delta n^{2}\right\rangle(\Delta t)=\left\langle\sum_{n_{0}} \rho_{n_{0}}\left(t_{0}\right) \sum_{n} \rho_{n}\left(t_{0}+\Delta t\right)\left(n-n_{0}\right)^{2}\right\rangle
$$

where $\rho_{n_{0}}\left(t_{0}\right)$ describes the initial site distribution. This equation corresponds to Eq. (15) of the main text (with a slightly different notation, where $n_{0} \rightarrow n, n \rightarrow n^{\prime}$, and the inverse number of ensemble members $M^{-1}$ appears to match the notation of Eq. (14)).

At the initial time $t=t_{0}(\Delta t=0)$, the above expression yields twice the variance of the initial state,

$$
\left\langle\Delta n^{2}\right\rangle(\Delta t=0)=2\left\langle\sum_{n_{0}} \rho_{n_{0}}\left(t_{0}\right)\left(n_{0}-\bar{n}\left(t_{0}\right)\right)^{2}\right\rangle
$$


In the graph shown in Figure 5 of the main text, this term was subtracted in order to match the zero-time MSD with the simpler expression Eq. (14).

\section{References}

[1] R. Binder, D. Lauvergnat, I. Burghardt, Phys. Rev. Lett. 2018, 120, 227401.

[2] B. Kuei, E. D. Gomez, Soft Matter 2017, 13, 49.

[3] B. McCulloch, V. Ho, M. Hoarfrost, C. Stanley, C. Do, W. T. Heller, R. A. Segalman, Macromolecules 2013, 46, 1899-1907.

[4] J. Gross, M. Ivanov, W. Janke, J. Phys.: Conf. Ser. 2016, 750, 012009.

[5] D. Lauvergnat, A. Nauts, J. Chem. Phys. 2002, 116, 8560.

[6] R. Binder, S. Römer, J. Wahl and I. Burghardt, J. Chem. Phys. 2014, 141, 014101.

[7] R. Binder and I. Burghardt, Faraday Discuss. 2020, 221, 406.

[8] R. Binder, M. Bonfanti, D. Lauvergnat, and I. Burghardt, J. Chem. Phys. 2020, 152, 204119 . 